\section{RSP}

http://www.rsp.fsp.usp.br/
Revista de Saúde Pública

\title{
Gastos públicos com internações hospitalares para tratamento da covid-19 no Brasil em 2020
}

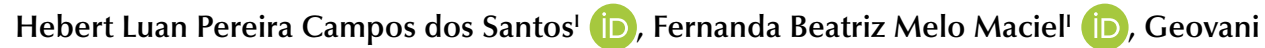 \\ Moreno Santos Junior" iD, Poliana Cardoso Martins" iD, Nília Maria de Brito Lima Prado" iD \\ I Universidade Federal da Bahia. Instituto Multidisciplinar em Saúde - Campus Anísio Teixeira. Vitória da \\ Conquista, BA, Brasil \\ " Universidade Federal da Bahia. Instituto Multidisciplinar em Saúde - Campus Anísio Teixeira. Programa de \\ Pós-Graduação em Saúde Coletiva. Vitória da Conquista, BA, Brasil
}

\section{Correspondência:}

Nília Maria de Brito Lima Prado Instituto Multidisciplinar em Saúde/Campus Anísio Teixeira Universidade Federal da Bahia (UFBA) R. Hormindo Barros 58, Quadra 17, Lote 58, Candeias

45029-094 Vitória da Conquista, BA, Brasil

E-mail: nilia.prado@ufba.br

Recebido: 15 mar 2021

Aprovado: 7 jul 2021

Como citar: Santos HLPC, Maciel FBM, Junior GMS, Martins PC, Prado NMLB. Gastos públicos com internações hospitalares para tratamento da covid-19 no Brasil em 2020. Rev Saude Publica. 2021;55:52.

https://doi.org/10.11606/ s1518-8787.2021055003666

Copyright: Este é um artigo de acesso aberto distribuído sob os termos da Licença de Atribuição Creative Commons, que permite uso irrestrito, distribuição e reprodução em qualquer meio desde que o autor e a fonte originais sejam creditados.

\section{RESUMO}

OBJETIVO: Descrever os gastos decorrentes das internações para tratamento clínico de usuários diagnosticados com Covid-19 no Sistema Único de Saúde (SUS) entre fevereiro e dezembro de 2020.

MÉTODOS: Estudo descritivo, elaborado a partir de dados do Sistema de Informações Hospitalares sobre gastos governamentais com internações hospitalares para tratamento clínico de usuários diagnosticados com Covid-19 e das causas incluídas nos capítulos do CID-10. Obteve-se o número de internações, tempo médio de permanência, taxa de letalidade e os gastos totais considerando serviços hospitalares, serviços profissionais e gasto médio por internação.

RESULTADOS: No período avaliado, ocorreram 462.149 internações hospitalares no SUS, sendo 4,9\% delas para tratamento de usuários com coronavírus. O gasto total foi superior a 2,2 bilhões de reais, sendo $85 \%$ destinados a serviços hospitalares e $15 \%$ a serviços profissionais. Os gastos para o tratamento da covid-19 se distribuíram de forma distinta entre as regiões do país. A região Sudeste teve o maior número de internações, maior valor total gasto, maior média de permanência em dias e maior taxa de letalidade, enquanto a região Sul registrou maior porcentagem de gastos com hospitais sem fins lucrativos (58\%) e hospitais empresariais (15\%).

CONCLUSÕES: As internações para tratamento clínico da infecção pelo coronavírus foram mais onerosas se comparadas às internações para tratamento de insuficiências respiratórias agudas e pneumonias ou influenza. Os resultados demonstraram as disparidades frente aos gastos de internação para procedimentos similares entre as regiões do país, evidenciando a vulnerabilidade e a necessidade de estratégias para diminuir as diferenças no acesso, uso e distribuição de recursos do SUS, garantindo equanimidade, e considerando as injustas desigualdades entre as regiões do país.

DESCRITORES: Infecções por Coronavirus. Hospitalização. Custos Hospitalares. Gastos Públicos com Saúde. Sistema Único de Saúde. 


\section{INTRODUÇÃO}

A infecção pelo SARS-CoV-2 vem se apresentando como um dos maiores desafios para os sistemas de saúde. Desde seu surgimento, em dezembro de 2019 em Wuhan, na China, a doença causada pelo novo coronavírus já somava mais de 161.513 .458 casos confirmados e 3.352.109 mortes em todo o mundo, até 15 de maio de $2021^{1}$. Clinicamente, a infecção pode se manifestar de três formas distintas, que variam de acordo a faixa etária e condições clínicas associadas: portador assintomático, indivíduo com doença respiratória aguda (DRA) ou usuários com pneumonia em diferentes graus de gravidade ${ }^{2}$.

A covid-19 compõe o grupo das infecções respiratórias agudas (IRA), síndromes clínicas cujos agentes infecciosos mais comuns são os vírus respiratórios, como o sincicial, ou bactérias, como Streptococcus pneumoniae e Haemophilus influenzae $e^{3}$. A gravidade da doença vai depender do patógeno causador da infecção, do meio ambiente e de fatores do hospedeiro. Dessa forma, de acordo com a Organização Mundial da Saúde (OMS), as IRA estão entre as doenças infecciosas com os maiores índices de morbimortalidade em todo o mundo ${ }^{4}$. Os dados apontam que essas infecções são responsáveis por mais de 4 milhões de mortes por ano, representando uma das principais causas de óbito nos países em desenvolvimento ${ }^{5}$.

No Brasil, em períodos anteriores à pandemia, tais infecções chegaram a ser responsáveis por cerca de um quinto das internações hospitalares do Sistema Único de Saúde (SUS) ${ }^{6}$. Só a Covid-19, até 15 de maio de 2021, registrou mais de 432 mil mortes e 15.519 .525 infectados, mas seus impactos no setor saúde e na economia nacional começaram a ser percebidos ainda no início de $2020^{7,8}$. Cabe destacar que o Brasil é um dos poucos países das Américas a contar com um sistema de saúde universal e gratuito, do qual cerca de $75 \%$ da população depende exclusivamente, e que é reconhecido como uma das políticas públicas mais importantes do país.

O SUS é citado como exemplo de sistema de saúde bem-sucedido na América Latina, principalmente devido à garantia de saúde como um direito de todos os cidadãos brasileiros. Por outro lado, os desafios do subfinanciamento têm colocado em risco a garantia desse direito ${ }^{10}$. No contexto da pandemia, o papel desempenhado pelo SUS é notório, especialmente, na atenção hospitalar. A Covid-19 tem demandado taxas adicionalmente altas de hospitalização" ${ }^{11}$, e para além da atenção à pressão gerada sobre o sistema de saúde, cabe observar e analisar os gastos representados para o SUS e como são distribuídos pelo território.

No cenário da pandemia da Covid-19, o desenvolvimento de análises que permitam estimar os custos diretos médico-hospitalares demandados, envolvendo exames, procedimentos, medicamentos, recursos humanos, consultas, internações, reabilitação e outros ${ }^{12}$, torna-se indispensável para subsidiar a tomada de decisão, no que concerne ao atendimento das demandas nos serviços e ao desenvolvimento de estratégias operacionais pelos sistemas nacionais de saúde ${ }^{13}$.

Em pesquisa na Biblioteca Virtual em Saúde (BVS), Scientific Electronic Library Online (SciELO) e na PubMed, utilizando os Descritores em Ciências da Saúde (DEcS/Mesh), nos idiomas inglês e português, "custos hospitalares", "gastos em saúde”, "gastos públicos com saúde", "despesas públicas" e "gastos públicos com serviços privados" associado aos termos "covid-19" e "Brazil", através do operador boleano AND, não foram encontrados estudos anteriores a este, de abrangência nacional, que descrevessem os custos para o SUS decorrentes das internações hospitalares para tratamento clínico de usuários confirmados para covid-19. Depreende-se que pesquisas referentes a esses gastos ainda são incipientes na literatura, embora a abordagem dessa temática seja de extrema relevância para compreender os custos ao sistema público de saúde.

Dessa forma, o objetivo do presente estudo foi analisar as proporções dos gastos públicos com internações para tratamento clínico de usuários internados com xcovid-19 pelo SUS no Brasil, entre os meses de fevereiro e dezembro de 2020. Para isso, foram descritos e 
analisados os gastos públicos com as internações hospitalares e como estão distribuídos em relação às características da população, podendo colaborar para a adoção de medidas capazes de evitar complicações e, consequentemente, o colapso do sistema de saúde no cenário da pandemia.

\section{MÉTODOS}

Trata de um estudo descritivo, a partir de dados secundários sobre gastos governamentais com internações hospitalares para tratamento clínico de usuários diagnosticados com covid-19 no Brasil no período de fevereiro a dezembro de 2020.

Os dados sobre as internações e seus respectivos custos foram coletados a partir do Sistema de Informações Hospitalares do Sistema Único de Saúde (SIH-SUS), disponibilizado no site do Departamento de Informática do SUS (Datasus) ${ }^{14}$, mediante informações consolidadas das Autorizações de Internação Hospitalar (AIH), classificados por região do país.

As internações selecionadas tinham como procedimento principal o código 03.03.01.022-3 (tratamento de infecção pelo coronavírus) - que corresponde às ações necessárias para o tratamento clínico do usuário internado com diagnóstico de infecção pelo coronavírus, conforme a Portaria no 245 , de 24 de março de 2020, e de acordo com as Orientações técnicas para operacionalização do SIH-SUS durante o estado de emergência de saúde pública por coronavírus, atualizadas pelo Ministério da Saúde em 13 de Agosto de $2020^{15}$-, nas unidades hospitalares vinculadas ao SUS (públicas e privadas conveniadas).

Paralelamente, buscou-se comparar os gastos com internações hospitalares para tratamento de paciente diagnosticado com Covid-19, gastos com internações por todas as causas considerando os capítulos do CID-10 e gastos com tratamento das principais infecções do sistema respiratório, tais quais pneumonias, gripe e infecções agudas das vias áreas superiores e inferiores. Para essas, selecionou-se os procedimentos com código: 03.03.14.015-1 tratamento de pneumonias ou influenza (gripe), 03.03.14.010-0 - tratamento de infecções agudas das vias aéreas superiores e 03.03.14.014-3 - tratamento de outras infecções agudas das vias aéreas inferiores ${ }^{16}$.

O estudo não considerou a utilização de tratamentos sem comprovação científica para infecção pelo coronavírus. A internação para o tratamento compreende ações e procedimentos necessários para estabilizar e evitar a piora do quadro clínico do usuário. Porém, tais intervenções podem variar substancialmente a depender do quadro clínico de cada usuário (considerando as comorbidades associadas e grau de comprometimento pulmonar), entre os protocolos clínicos adotados por cada estado e município e entre as práticas clínicas assumidas.

O recorte temporal escolhido compreende os meses de fevereiro a dezembro de 2020 para as internações para tratamento clínico de usuários diagnosticados com coronavírus. A delimitação desse horizonte temporal justifica-se, pois em 26 de fevereiro de 2020 foi confirmado o primeiro caso da infecção pelo SarS-CoV-2 no Brasil, e por dezembro constituir o último mês em que foram disponibilizados os dados no SIH-SUS.

As variáveis de interesse do estudo retiradas do SIH-SUS compreenderam: número de internações, valor total das internações, valor dos serviços profissionais, valor dos serviços hospitalares, valor médio de internação (valor médio com internação hospitalar no SUS, por especialidade ou procedimento, em determinado espaço geográfico, no ano considerado), média de permanência (média do total de dias de internação referentes às AIH aprovadas no período) e taxa de letalidade (razão entre a quantidade de óbitos e o número de AIH aprovadas, computadas como internações, no período, multiplicada por $100)^{17}$. Para os gastos, considerou-se os valores em reais, e a média de permanência foi contabilizada em dias. 
Além disso, buscou-se identificar os valores totais pagos por esfera jurídica, ou seja, a distribuição dos gastos totais governamentais de acordo com a esfera jurídica, considerando as categorias do Cadastro Nacional de Estabelecimentos de Saúde e: "hospitais de administração pública”, "hospitais empresariais" e "hospitais sem fins lucrativos". O primeiro grupo agrega aqueles que têm como esfera jurídica "administração pública federal", "estadual ou Distrito Federal", "municipal" e "administração pública - outros". Os empresariais são aqueles que têm como esfera jurídica "empresa pública ou sociedade de economia mista" e "demais entidades empresariais". Já os hospitais sem fins lucrativos são os cadastrados com a esfera jurídica "entidades sem fins lucrativos" ${ }^{18}$.

Para as tabulações de número, proporção, razão e valores pagos pelas internações, oriundos do SIH-SUS, utilizou-se o programa Microsoft Excel e procedeu-se análise estatística descritiva.

A análise foi realizada sobre dados secundários, de acesso público, sem possibilidade de identificação individual das informações. Assim, consoante as recomendações da Resolução do Conselho Nacional de Saúde (CNS) no 466, de 12 de dezembro de 2012, foram respeitados os princípios éticos de pesquisas que envolvem seres humanos, sendo dispensada a aprovação do comitê de ética em pesquisa.

\section{RESULTADOS}

Entre fevereiro e dezembro de 2020, foram registrados no SIH-SUS 462.149 internações hospitalares que tiveram como procedimento principal tratamento de usuários com coronavírus, correspondendo a um gasto total de $\mathrm{R} \$ 2.248 .011 .968,40$. Cabe considerar que $85 \%$ desse valor foi gasto com serviços hospitalares e $15 \%$ com serviços profissionais. Dentre todas as regiões do país, verificou-se que a maior parcela dos gastos ocorreu na região Sudeste, representando cerca de $45 \%$ do total. As regiões Sul e região Norte apresentaram, respectivamente, o maior e o menor valor médio por internação (Tabela 1).

Ao considerar todas as internações ocorridas no Brasil no âmbito do SUS, no período analisado, 4,9\% tiveram como procedimento principal o tratamento de usuários com coronavírus, correspondendo a $15 \%$ dos gastos com todas as internações no país. Ao comparar a média de permanência em dias entre todas as internações ocorridas e as internações ocorridas para tratamento do coronavírus, verificou-se que as internações para tratamento do coronavírus duraram mais tempo (Tabela 2).

Tabela 1. Gastos (em reais) das internações hospitalares para tratamento clínico da infecção pelo

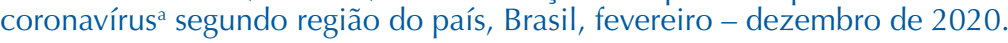

\begin{tabular}{|c|c|c|c|c|}
\hline Região & Valor total do gasto $(\mathrm{R} \$)$ & $\begin{array}{l}\text { Valor dos serviços } \\
\text { hospitalares }^{\mathrm{c}}(\mathbf{R} \$)\end{array}$ & $\begin{array}{l}\text { Valor dos serviços } \\
\text { profissionais }^{\text {d }}(\mathbf{R} \$)\end{array}$ & $\begin{array}{l}\text { Valor médio por } \\
\text { internação }(R \$)\end{array}$ \\
\hline Norte & $133.723 .986,43$ & $111.708 .340,36$ & $22.015 .646,07$ & $3.157,96$ \\
\hline Nordeste & $550.997 .791,12$ & $465.375 .038,13$ & $85.622 .752,99$ & $4.489,91$ \\
\hline Sudeste & $1.010 .529 .436,45$ & $857.747 .748,55$ & $152.776 .809,87$ & $5.154,34$ \\
\hline Sul & $381.184 .490,20$ & $324.887 .362,21$ & $56.297 .127,99$ & $6.165,64$ \\
\hline Centro-Oeste & $171.576 .264,24$ & $145.114 .948,61$ & $26.460 .690,51$ & $4.376,16$ \\
\hline Total & $2.248 .011 .968,44$ & $1.904 .833 .437,86$ & $343.173 .027,43$ & $4.864,26$ \\
\hline
\end{tabular}

Fonte: Elaboração própria a partir dos dados do SIH-SUS.

a Internações hospitalares de pacientes com covid-19 registradas com o procedimento código 03.03.01.022-3, conforme orientação da Portaria n ${ }^{\circ}$ 245, de 24 de março de 2020 e Orientações técnicas para operacionalização do SIH-SUS durante o estado de emergência de saúde pública por coronavírus publicado em 13 de agosto de 2020 pelo Ministério da Saúde.

${ }^{\mathrm{b}}$ Valor referente às $\mathrm{AlH}$ aprovadas no período. Este valor não obrigatoriamente corresponde ao valor repassado ao estabelecimento, pois, dependendo da situação das unidades, estes recebem recursos orçamentários ou pode haver retenções e pagamentos de incentivos, não aqui apresentados. Portanto, este valor deve ser considerado como o valor aprovado da produção.

${ }^{\mathrm{C}}$ Valor dos serviços hospitalares referente às $\mathrm{AlH}$ aprovadas no período.

${ }^{\mathrm{d}}$ Valor dos serviços profissionais referente às $\mathrm{AIH}$ aprovadas no período.

e Valor médio com internação hospitalar no Sistema Único de Saúde (SUS), por especialidade, em determinado espaço geográfico, no período considerado. 
Comparando os gastos e as internações que tiveram como procedimento principal tratamento de infecções agudas, de vias aéreas superiores e inferiores, ou de pneumonia ou influenza, com internações para tratamento do coronavírus, os maiores gastos e número de internações também remetem à infecção pelo coronavírus (Figura 1).

Tabela 2. Gastos (em R\$) e média de permanência (em dias) das internações hospitalares para Capítulos CID-10 X internações para tratamento clínico da infecção pelo coronavírus segundo região do país, Brasil, fevereiro - dezembro de 2020.

\begin{tabular}{|c|c|c|c|c|c|c|c|c|c|c|}
\hline \multirow[b]{2}{*}{ Região } & \multicolumn{4}{|c|}{ Internações para Capítulos CID-10 ${ }^{a}$} & \multicolumn{6}{|c|}{ Internações para tratamento da infecção pelo coronavírus ${ }^{\text {b }}$} \\
\hline & Internações & $\begin{array}{l}\text { Valor total do } \\
\text { gasto }^{c}(\mathrm{R} \$)\end{array}$ & $\begin{array}{c}\text { Valor } \\
\text { médio por } \\
\text { internação }\end{array}$ & $\begin{array}{c}\text { Média de } \\
\text { permanência } \\
{\text { (em dias })^{f}}^{\text {(em }}\end{array}$ & Internações & $\begin{array}{c}\%^{\text {e do }} \\
\text { total de } \\
\text { internações }\end{array}$ & $\begin{array}{l}\text { Valor total do } \\
\text { gasto }^{c}(\mathbf{R} \$)\end{array}$ & $\begin{array}{l}\text { \% do total } \\
\text { de valores } \\
\text { gastos com } \\
\text { internações }\end{array}$ & $\begin{array}{c}\text { Valor } \\
\text { médio por } \\
\text { internação }^{d}\end{array}$ & $\begin{array}{c}\text { Média de } \\
\text { permanência } \\
\text { (em dias) }^{\text {f }}\end{array}$ \\
\hline Norte & 758.143 & $819.819 .970,59$ & $1.081,35$ & 4,7 & 42.345 & 5,6 & $133.723 .986,4$ & 16,3 & $3.157,96$ & 7,4 \\
\hline Nordeste & 2.461 .730 & $3.462 .075 .135,95$ & $1.406,36$ & 5,3 & 122.719 & 5,0 & $550.997 .791,1$ & 15,9 & $4.489,91$ & 7,8 \\
\hline Sudeste & 3.772 .966 & $6.511 .672 .543,21$ & $1.725,88$ & 5,9 & 196.054 & 5,3 & 1.010 .529 .436 & 15,5 & $5.154,34$ & 8,6 \\
\hline Sul & 1.646 .796 & $3.055 .201 .666,37$ & $1.855,24$ & 5,2 & 61.824 & 3,7 & $381.184 .490,2$ & 12,5 & $6.165,64$ & 8,5 \\
\hline Centro-Oeste & 746.075 & $1.064 .013 .109,82$ & $1.426,15$ & 5,1 & 39.207 & 5,2 & $171.576 .264,2$ & 16,1 & $4.376,16$ & 7,4 \\
\hline Total & 9.385.710 & $14.912 .782 .425,94$ & $1.588,88$ & 5,4 & 462.149 & 4,9 & 2.248.011.968,44 & 15 & $4.864,26$ & 8,2 \\
\hline
\end{tabular}

Fonte: Elaboração própria a partir dos dados do SIH-SUS.

a Todas as internações ocorridas no âmbito do SUS considerando todos os capítulos do CID-10.

b Todas as internações ocorridas no âmbito do SUS com o procedimento código 03.03.01.022-3, conforme orientação da Portaria n 245 , de 24 de março de 2020 e Orientações técnicas para operacionalização do SIH-SUS durante o estado de emergência de saúde pública por coronavírus publicado em 13 de agosto de 2020 pelo Ministério da Saúde.

' Valor referente às AIH aprovadas no período. Este valor não obrigatoriamente corresponde ao valor repassado ao estabelecimento, pois, dependendo da situação das unidades, estes recebem recursos orçamentários ou pode haver retenções e pagamentos de incentivos, não aqui apresentados. Portanto, este valor deve ser considerado como o valor aprovado da produção.

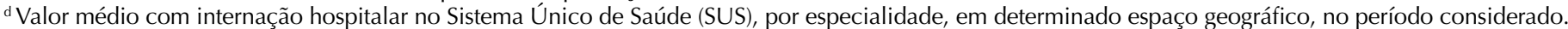

e As porcentagens foram calculadas tomando como base os valores das internações e gastos totais por região para todos os capítulos do CID-10.

${ }^{\mathrm{f}}$ Média de permanência das internações referentes às AIH aprovadas, computadas como internações, no período.

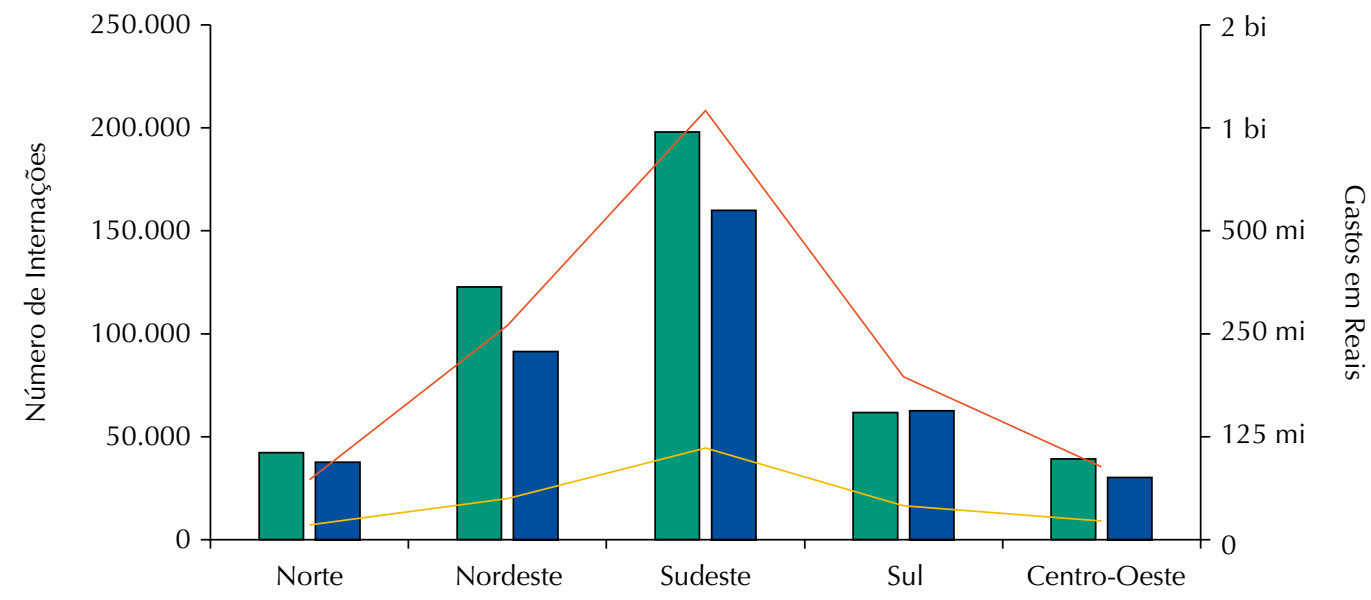

Internações para tratamento de pneumonias e IRA

$\square$ Internações para tratamento da infecção pelo coronavírus

Gastos das internações para tratamento de pneumonias e IRA

- Gastos das internações para tratamento da infecção pelo coronavírus

a Procedimento com código 03.03.01.022-3 (tratamento de infecção pelo coronavírus) - que corresponde às ações necessárias para o tratamento clínico do paciente internado com diagnóstico de covid-19.

b Procedimentos com códigos 03.03.14.015-1 - tratamento de pneumonias ou influenza (gripe),

03.03.14.010-0 - tratamento de infecções agudas das vias aéreas superiores e 03.03.14.014-3 - tratamento de outras infecções agudas das vias aéreas inferiores.

IRA: infecções respiratórias agudas.

Figura 1. Internações hospitalares e gastos para tratamento da infecção por coronavírus ${ }^{\mathrm{a}}$ e para tratamento de infecções agudas de vias aéreas inferiores e superiores, pneumonia ou influenzab por região do país, Brasil, fevereiro - dezembro de 2020. 


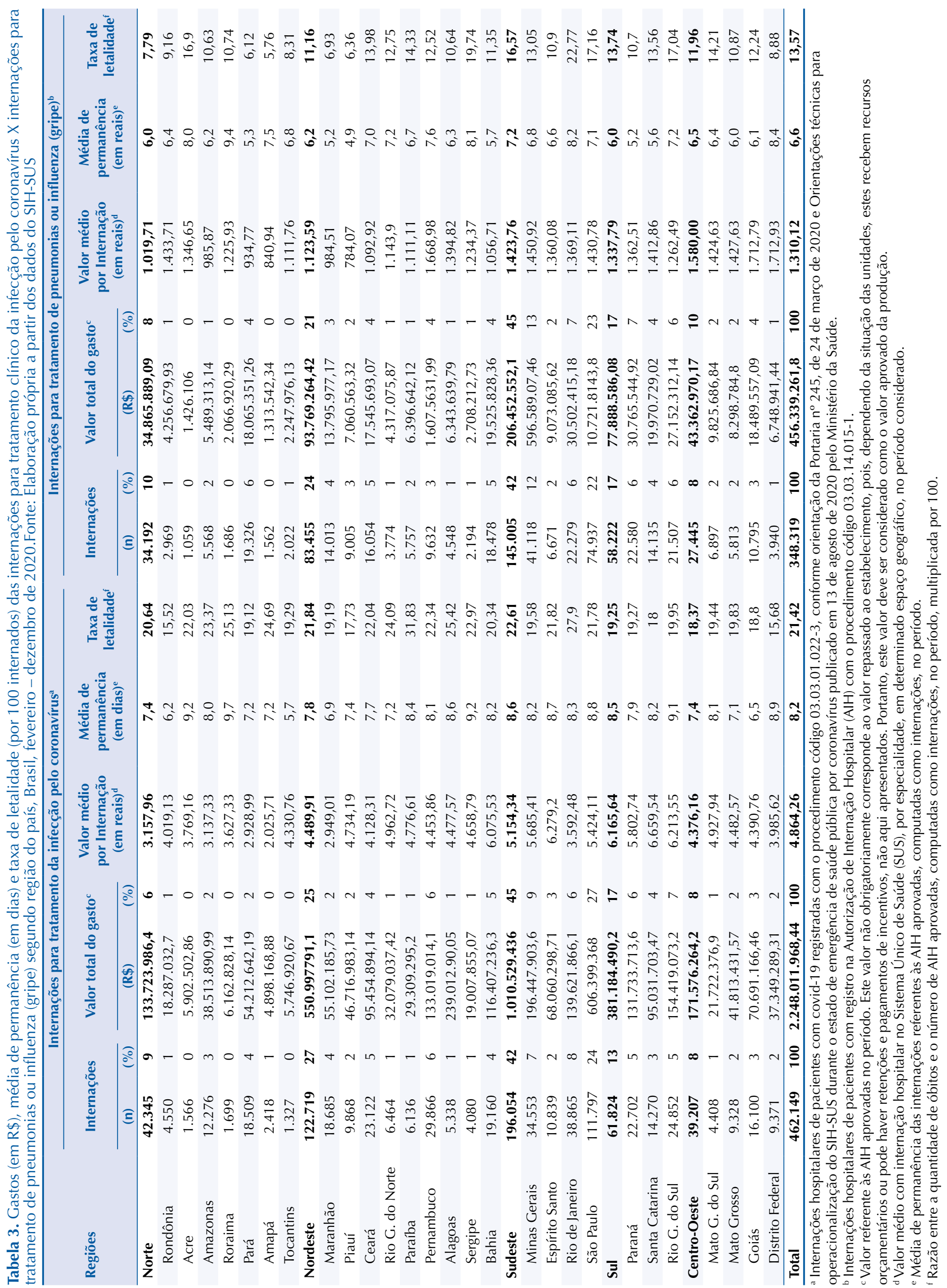




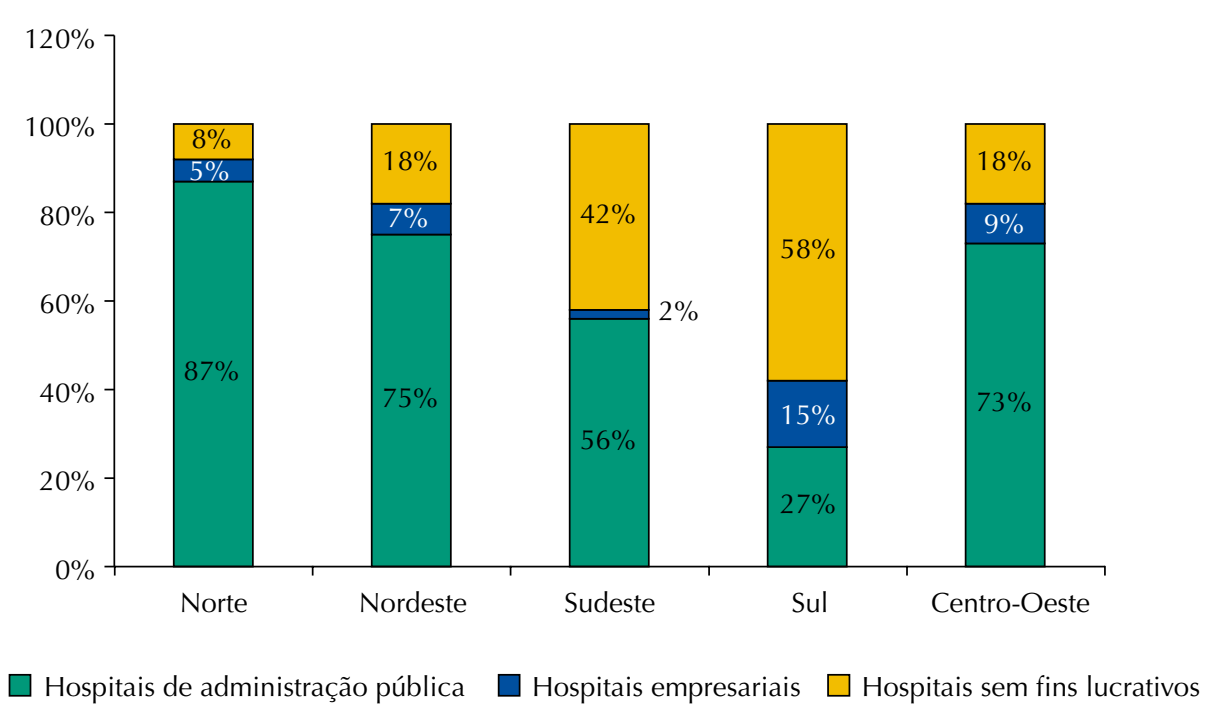

Fonte: Elaboração própria a partir dos dados do SIH-SUS.

Figura 2. Distribuição dos gastos das internações hospitalares para tratamento clínico da infecção por coronavírus entre as esferas jurídicas dos hospitais do SUS por região do país, Brasil, fevereiro dezembro de 2020.

Quanto aos gastos associados à média de permanência e à taxa de letalidade, comparando internações para tratamento da Covid-19 a internações para tratamento de pneumonia ou influenza, a região Sudeste apresentou o maior número de internações, maior valor total gasto, maior média de permanência em dias e maior taxa de letalidade. Os estados de São Paulo e Minas Gerais apresentaram os maiores gastos para tratamento dessas condições. Já o maior tempo de permanência para tratamento, tanto da infecção pelo coronavírus como para tratamento de pneumonia ou influenza, foi identificado no estado de Roraima. Os estados da Paraíba e Rio de Janeiro apresentaram as maiores taxas de letalidade, respectivamente, relacionadas a tratamento do coronavírus e tratamento da influenza ou gripe (Tabela 3).

No que diz respeito à porcentagem de distribuição dos gastos totais das internações para tratamento da infecção pelo coronavírus entre as esferas jurídicas dos hospitais do SUS, verificou maior porcentagem de gastos com hospitais sem fins lucrativos (58\%) e hospitais empresariais (15\%) no Sul do país (Figura 2).

\section{DISCUSSÃO}

No presente estudo, os resultados evidenciaram que os gastos públicos das internações hospitalares para tratamento da Covid-19 se distribuíram de forma distinta entre as regiões do país.

Considerando o volume de internações para tratamento clínico de usuários infectados pelo coronavírus no período analisado, fica evidente o impacto que tais internações geram no sistema de saúde. Embora as internações pagas pelo SUS não representem a totalidade dos casos que necessitam de internação hospitalar, o caráter de evolução progressiva com o qual se desdobra o quadro clínico do infectado confere grande cobertura de assistência ao SIH-SUS para essas internações.

As variações geográficas em torno do valor médio pago por internação no país, constatadas pelas diferenças significativas nos gastos regionais, independentemente da situação pandêmica da Covid-19, também foram relatadas em outros estudos ${ }^{20}$. Nota-se que o custo médio da internação é maior para esse tratamento quando comparado ao de todas as causas de internação e, mais especificamente, quando comparado aos casos de tratamento de pneumonias ou influenza. Tal fato permite afirmar que as internações para tratamento clínico da Covid-19 são mais onerosas do que aquelas por outras causas. Cabe considerar 
que as variações entre o valor médio da internação e a média de permanência decorrem de fatores socioeconômicos, epidemiológicos e demográficos, que determinam o perfil da demanda hospitalar pelo SUS, além de políticas públicas de atenção à saúde ${ }^{21}$.

Ante esse cenário, somam-se as medidas de austeridade fiscal e a forte restrição de receitas pelo nível federal, que são a principal fonte de recursos para a execução dos serviços no SUS. Ainda que tenha sido regulamentada a transferência de recursos financeiros federais específicos para os estados e municípios custearem ações e serviços públicos relacionadas ao enfrentamento da Covid-19, as mudanças normativas recentes no financiamento das ações, com ausência de discricionariedade na aplicação dos recursos pelos entes federados estaduais e municipais, têm intensificado a desigualdade regional na oferta de ações e serviços pelo SUS, inclusive ambulatoriais e hospitalares. Com a aprovação, em 2016, da Emenda Constitucional no 95, que estabeleceu um teto de gastos para o Governo Federal, o SUS perdeu quase 22,5 bilhões de reais entre 2018 e $2020^{22}$. Nesse aspecto, a pandemia impôs novos desafios ao sistema, em virtude das características de transmissibilidade comunitária da doença ${ }^{23}$ e, como demonstrado pelos dados aqui apresentados, do custo mais elevado das internações.

Sabe-se que a maioria dos usuários com Covid-19 evolui com poucos sintomas ou até mesmo assintomática. No entanto, de acordo com a $\mathrm{OMS}^{24}$, cerca de $14 \%$ dos casos identificados desenvolvem doença grave, exigindo internação hospitalar e oxigenoterapia, $5 \%$ necessitam de internação em Unidades de Terapia Intensiva (UTI), e boa parte dos internados em UTI demandam suporte ventilatório ${ }^{24}$.

Para a análise dos dados e tomada de decisões, é importante considerar a distribuição heterogênea, entre as regiões do país, da estrutura etária e mobilidade da população, do percentual de pessoas em situação de vulnerabilidade e de prevalência de comorbidades, pois são fatores que podem determinar quadros mais graves da infecção pelo Sars-CoV-2. Soma-se a esse cenário os efeitos das medidas de enfrentamento aplicadas por cada estado, as quais têm relação direta com a incidência de $\operatorname{casos}^{25}$.

As desigualdades em relação aos gastos públicos para tratamento clínico da Covid-19 observadas neste estudo remetem aos diferentes cenários, do ponto de vista assistencial e epidemiológico, encarados pelas regiões brasileiras e que constituem diferenças históricas na capacidade e cobertura do sistema de saúde. Nesta pesquisa, a região Sudeste apresentou o maior número de internações e o maior gasto total para internações para tratamento da infecção pelo coronavírus. Possíveis explicações estão associadas aos maiores números de infectados concentrados nessa região e a maior disponibilidade de oferta de leitos ${ }^{11}$. Apesar disso, em um país marcado por desigualdades, inclusive, quanto à oferta de serviços de saúde, Noronha et al. ${ }^{11}$ atentam para a presença de vazios assistenciais, que pode levar sistemas locais ao colapso.

A análise da letalidade entre internados para tratamento clínico da Covid-19 aponta que 4 dos 5 estados do Sudeste, 4 dos 7 estados da região Norte e 6 dos 9 estados do Nordeste ultrapassaram a letalidade média do país, enquanto os demais estados apresentaram letalidade abaixo da média nacional. Aqueles que registraram maior taxa de letalidade (Paraíba, Alagoas e Roraima) e maiores tempos médios de permanência (Roraima, Sergipe e Acre) estão nas regiões Norte e Nordeste. Tais dados podem revelar, em alguma medida, desigualdades econômicas, sociais e assistenciais que, para serem sanadas, demandam políticas que levem em conta as singularidades regionais ${ }^{25}$.

A disponibilidade de recursos em determinadas regiões, por exemplo, de máquinas para diálise e equipamentos de ventilação não invasiva e invasiva, pode estar relacionada ao aumento dos custos das internações nas demais. Um mapeamento detalhado do número de leitos de UTI e de ventiladores e respiradores mostrou que o Brasil possui uma razão de 15,6 leitos de UTI por 100 mil habitantes, sendo a média no SUS de 7,1 e com enorme heterogeneidade regional, além da escassez de recursos na maioria das localidades ${ }^{20}$. 
Tais achados corroboram análises ${ }^{26,27}$ realizadas por pesquisadores brasileiros a respeito da relação entre os maiores níveis de vulnerabilidade à pandemia e uma combinação de infraestrutura de leitos de UTI aquém do mínimo, mortalidade por condições similares à covid-19 acima da mediana nacional ${ }^{20} \mathrm{e}$ acentuação dos efeitos da doença nas regiões mais pobres, como o Norte e Nordeste ${ }^{28}$.

No sistema de saúde brasileiro, a relação entre público e privado torna-se ainda mais complexa ao se analisar a participação do Estado no financiamento da saúde suplementar, no que concerne ao percentual pago pelo SUS a hospitais sem fins lucrativos e a hospitais empresariais na região Sul do país. Dessa forma, torna-se urgente ampliar a coordenação da oferta de leitos pelo SUS com a disponibilidade equitativa, para que determinadas áreas sejam priorizadas pelo investimento governamental diante da situação emergencial instaurada pela epidemia da covid-1929.

Entre os achados aqui apresentados, há dois resultados importantes. Em primeiro lugar, o impacto significativo que a covid-19, em termos de gastos públicos em saúde, representa para a carga de internações por doenças agudas do aparelho respiratório no país, haja vista o aumento dos gastos totais com internações no período analisado. Em segundo lugar, e talvez mais importante, as disparidades nos gastos com internação para procedimentos similares entre as regiões do país. Isso posto, cabe analisar as lacunas existentes no processo de padronização das práticas clínicas e assistenciais e elucidar as dificuldades e diferenças encontradas em cada localidade que justifiquem tais disparidades. Junto a isso, faz-se necessário compreender quais estratégias, a médio e longo prazo, são possivelmente implementáveis para diminuir as diferenças no acesso, uso e distribuição de recursos, visando reduzir o impacto financeiro nas contas do sistema público de saúde.

Algumas limitações deste estudo devem ser ressaltadas. A primeira refere-se ao fato de os dados dos últimos seis meses disponíveis pelo SIH-SUS estarem sujeitos a atualização. Não obstante, é o único sistema de informação hospitalar de domínio público do país ${ }^{19}$. A segunda diz respeito à avaliação da cobertura do sistema, que abrange apenas as internações realizadas na rede pública e conveniada ao SUS, excluindo internações realizadas de forma particular e por planos de saúde ${ }^{19}$. Ainda assim, considera-se um sistema importante para análise do perfil de morbidade, já que $80 \%$ do universo das internações hospitalares no Brasil são cobertas pelo SIH-SUS, variando conforme nível de complexidade e procedimento demandado ${ }^{19}$. A terceira limitação importante compreende a ausência de abordagem sobre o perfil dos usuários internados, considerando gênero, raça/cor, idade, comorbidades associadas, entre outros aspectos que poderiam auxiliar na avaliação da rede hospitalar do SUS durante a pandemia. A não realização dessas análises se deve à indisponibilidade de tais variáveis pelo SIH-SUS, ao trabalhar com dados consolidados das AIH.

Os dados disponíveis não permitem um recorte mais detalhado sobre as características dos usuários internados para tratamento do coronavírus. Além disso, há também o entendimento de que as estatísticas relacionadas à morbidade hospitalar apresentam restrições seletivas, fornecendo informações apenas dos usuários cujo quadro clínico demandou hospitalização, e restrições parciais, como usuários que precisariam de hospitalização, mas, por algum motivo, não se internaram ${ }^{19,30}$.

\section{CONCLUSÕES}

Os dados aqui apresentados referem-se a um recorte temporal delimitado, que permite avaliar o início da pandemia no Brasil. Identificou-se que os gastos públicos representados pelas internações para tratamento da infecção pelo coronavírus, entre os meses de fevereiro e dezembro de 2020, tiveram custo de mais de 2,2 bilhões de reais. Ademais, os resultados ora apresentados permitem inferir que as internações para tal fim foram mais onerosas se comparadas àquelas para tratamento de IRA e pneumonias ou influenza. 
Até onde sabemos, este é o primeiro estudo a estimar os custos das internações para tratamento da Covid-19 no SUS, e pode acrescentar reflexões necessárias ante o cenário de eminente colapso do sistema de saúde em quase todo o país. Novos estudos com análises de custos que considerem outras abordagens são necessários para monitorar o impacto econômico da pandemia sobre o sistema de saúde nacional a longo prazo, além da construção e execução de respostas que considerem características locais, sociais e demográficas. Nesse cenário, frente ao papel desempenhado pelo SUS para assegurar a vida de milhares de brasileiros durante a pandemia, a saúde não pode ser encarada como gasto, mas como investimento em defesa da vida do povo brasileiro.

\section{REFERÊNCIAS}

1. Organização Pan-Americana da Saúde. Folha informativa COVID-19-. Brasília, DF: OPAS-Brasil; 2021 [citado 12 fev 2021]. Disponível em: https://www.paho.org/pt/covid19

2. Xavier AR, Silva JS, Almeida JPCL, Conceição JFF, Lacerda GS, Kanaan S. COVID-19: manifestações clínicas e laboratoriais na infecção pelo novo coronavírus. J Bras Patol Med Lab. 2020;56(1):1-9. https://doi.org/10.5935/1676-2444.20200049

3. Cardoso AM. A persistência das infecções respiratórias agudas como problema de Saúde Pública. Cad Saude Publica. 2010;26(7):1270-1. https://doi.org/10.1590/S0102-311X2010000700001

4. World Health Organization. Pandemic and epidemic diseases. Infection prevention and control of epidemic and pandemic-prone acute respiratory infections: WHO guidelines. Geneva (CH): WHO; 2014 [citado 13 fev 2021]. Disponível em: http://apps.who.int/iris/ bitstream/10665/112656/1/9789241507134_eng.pdf?ua=1

5. Fórum das Sociedades Respiratórias Internacionais. Doenças respiratórias no mundo: realidades de hoje - oportunidades para o amanhã. Sheffield (UK): European Respiratory Society Publications Office; 2013 [citado 22 fev 2021]. Disponível em https://www.thoracic.org/about/ global-public-health/firs/resources/FIRS-in-Portuguese.pdf

6. Godoy DV, Dal Zotto C, Bellicanta J, Weschenfelder F, Nacif SB. Doenças respiratórias como causa de internações hospitalares de pacientes do Sistema Único de Saúde num serviço terciário de clínica médica na região nordeste do Rio Grande do Sul. J Pneumol. 2001;27(4):193-8. https://doi.org/10.1590/S0102-35862001000400005

7. Ministério da Saúde (BR). COVID-19: Painel Coronavirus Brasil. Brasília, DF; 2021 [citado 5 maio 2021]. Disponível em: https://covid.saude.gov.br/

8. Marinelli NP, Albuquerque LPA, Sousa IDB, Batista FMA, Mascarenhas MDM, Rodrigues MTP. Evolução de indicadores e capacidade de atendimento no início da epidemia de COVID-19 no Nordeste do Brasil, 2020. Epidemiol Serv Saude. 2020;29(3):e2020226. https://doi.org/10.5123/s1679-49742020000300008

9. Andrade RO. Covid-19 is causing the collapse of Brazil's national health service. BMJ. 2020;370:m3032. https://doi.org/10.1136/bmj.m3032

10. Souza DO. O subfinanciamento do Sistema Único de Saúde e seus rebatimentos no enfrentamento da Covid-19. Physis Rev Saude Coletiva. 2020;30(3):1-6. https://doi.org/10.1590/S0103-73312020300313

11. Noronha KVMS, Guedes GR, Turra CM, Andrade MV, Botega L, Nogueira D, et al. Pandemia por COVID-19 no Brasil: análise da demanda e da oferta de leitos hospitalares e equipamentos de ventilação assistida segundo diferentes cenários. Cad Saude Publica. 2020;36(6):e00115320. https://doi.org/10.1590/0102-311x00115320

12. Rocha R, Rache B, Nunes L, Massuda A. Estimação de custos de hospitalizações em UTI por COVID-19 no SUS: limite inferior por cenários populacionais de infecção. São Paulo, SP: Instituto de Estudos para Políticas de Saúde; 2020 [citado 20 fev 2021]. (Nota Técnica; nº 2). Disponível em: https://ieps.org.br/wp-content/uploads/2020/03/NT2_Corona.pdf

13. Arredondo R. Why revisit your cost-accounting strategy. Healthc Financ Manage. 2014;68(7):68-73.

14. Ministério da Saúde (BR), Departamento de Informática do Sistema Único de Saúde. Sistema de Informação Hospitalar Descentralizado - SIHD. Brasília, DF: DATASUS; c2016 [citado 12 fev 2021]. Disponível em: http://sihd.datasus.gov.br/principal/index.php 
15. Ministério da Saúde (BR), Secretaria de Atenção Especializada à Saúde. Portaria № 245 de 24 de março de 2020. Inclui procedimento na Tabela de Procedimentos, Medicamentos, Órteses, Próteses e Materiais Especiais do SUS, para atendimento exclusivo de pacientes com diagnóstico clínico de COVID-19 e altera o Sistema de Informação Hospitalar do SUS (SIH/SUS) para permitir o registro de ações relativas ao enfrentamento da COVID-19. Brasília, DF; 2020 [citado 12 fev 2021]. Disponível em: http://bvsms.saude.gov.br/bvs/saudelegis/saes/2020/ prt0245_15_06_2020_rep.html

16. Secretaria de Saúde do Estado de São Paulo. Orientações AIH Covid-19. São Paulo, SP; 2020 [citado 21 fev 2021]. (Circular; no 320/2020). Disponível em: http://www.fehosp.com.br/ files/circulares/5003fad52d33fc622273f3e3b911298b.pdf

17. Ministério da Saúde (BR), Departamento de Informática do Sistema Único de Saúde. Informações de Saúde: morbidade hospitalar do SUS por local de residência. Notas Técnicas. Brasília, DF: DATASUS; s.d. [citado 10 fev 2021]. Disponível em: http://tabnet.datasus.gov.br/ cgi/sih/nrdescr.htm

18. Ministério da Saúde (BR), Departamento de Informática do Sistema Único de Saúde. Informações de Saúde: Cadastro Nacional de Estabelecimentos de Saúde CNES. Notas Técnicas. Brasília, DF: DATASUS; s.d. [citado 10 fev 2021]. Disponível em: http://tabnet.datasus.gov.br/ cgi/cnes/NT_RecursosF\%C3\%ADsicos.htm

19. Lessa FJD, Mendes ACG, Farias SF, Sá DA, Melo Filho DA. Novas metodologias para vigilância epidemiológica: uso do Sistema de Informações Hospitalares - SIH/SUS. Inf Epidemiol SUS. 2000;9 Supl 1:3-27. https://doi.org/10.5123/S0104-16732000000500001

20. Rache B, Rocha R, Nunes L, Spinola P, Malik AM, Massuda A. Necessidades de infraestrutura do SUS em preparo ao COVID-19: leitos de UTI, respiradores e ocupação hospitalar. São Paulo, SP: Instituto de Estudos para Políticas de Saúde; 2020 [citado 20 fev 2021]. (Nota Técnica; nº 3). Disponível em: https://ieps.org.br/pesquisas/necessidades-de-infraestrutura-do-sus-em-preparoao-covid-19-leitos-de-uti-respiradores-e-ocupacao-hospitalar/

21. REDE Interagencial de Informação para a Saúde Indicadores e Dados Básicos para a Saúde no Brasil. Brasília, DF: OPAS-Brasil; 2008 [citado 20 fev 2021]. Capítulo 3, Fichas de Qualificação de Indicadores -E- Recursos: valor médio pago por internação hospitalar no SUS (AIH); p. 256. Disponível em: https://www.paho.org/bra/dmdocuments/indicadores.pdf

22. Menezes APR, Moretti BR, Reis AAC. O futuro do SUS: impactos das reformas neoliberais na saúde pública - austeridade versus universalidade. Saude Debate. 2019;43 № Espec 5:58-70. https://doi.org/10.1590/0103-11042019S505

23. Fernandes GAAL, Pereira BLS. Os desafios do financiamento do enfrentamento à COVID-19 no SUS dentro do pacto federativo. Rev Adm Publica. 2020;54(4):595-613. https://doi.org/10.1590/0034-761220200290

24. World Health Organization. Clinical management of severe acute interim guidance, 13 March 2020. Geneva (CH): WHO; 2020; [citado 22 fev 2021]. Disponível em: https://apps.who.int/iris/ handle/10665/331446

25. Cavalcante JR, Cardoso-dos-Santos AC, Bremm JM, Lobo AP, Macário EM, Oliveira WK, et al. COVID-19 no Brasil: evolução da epidemia até a semana epidemiológica 20 de 2020. Epidemiol Serv Saude. 2020;29(4):e2020376. https://doi.org/10.5123/s1679-49742020000400010

26. Rache B, Nunes L, Rocha R, Lago M, Fraga A. Como conter a curva no Brasil? Onde a epidemiologia e a economia se encontram. São Paulo, SP: Instituto de Estudos para Políticas de Saúde; 2020 [citado 26 fev 2021]. (Nota Técnica; nº 4). Disponível em: https://ieps.org.br/ pesquisas/como-conter-a-curva-no-brasil-onde-a-epidemiologia-e-a-economia-se-encontram/

27. Rache B, Rocha R, Nunes L, Spinola P, Massuda A. Para além do custeio: necessidades de investimento em leitos de UTI no SUS sob diferentes cenários da Covid-19. São Paulo, SP: Instituto de Estudos para Políticas de Saúde; 2020 [citado 26 fev 2021]. (Nota Técnica; n 7). Disponível em: https://ieps.org.br/pesquisas/para-alem-do-custeio-necessidades-de-investimentoem-leitos-de-uti-no-sus-sob-diferentes-cenarios-da-covid-19/

28. Kerr L, Kendall C, Silva EM, Pescarini JM, Almeida RLF, Ichihara MY, et al. COVID-19 no Nordeste brasileiro: sucessos e limitações nas respostas dos governos dos estados. Cienc Saude Coletiva. 2020;25 Supl 2:4099-120. https://doi.org/10.1590/1413-812320202510.2.28642020

29. Costa NRA. A disponibilidade de leitos em Unidade de Tratamento Intensivo no SUS e nos planos de saúde diante da epidemia da COVID-19 no Brasil. Rio de Janeiro, RJ: Observatorio Hospitalar Fiocruz; 2020 [citado 25 fev 2021]. Disponível em: http://observatoriohospitalar. fiocruz.br/sites/default/files/biblioteca/ESTUDO\%20NILSON_0.PDF 
30. Ranzani OT, Bastos LSL, Gelli JGM, Maechesi JF, Baião F, Hamacher S, et al. Characterisation of the first 250,000 hospital admissions for COVID-19 in Brazil: a retrospective analysis of nationwide data. Lancet Respir Med. 2021;9(4):407-18. https://doi.org/10.1016/S2213-2600(20)30560-9

Contribuição dos Autores: Concepção e planejamento do estudo: HLPCS, FBMM, GMSJ, PCM, NMLBP. Coleta, análise e interpretação dos dados: HLPCS, FBMM, GMSJ, PCM, NMLBP. Elaboração ou revisão do manuscrito: HLPCS, FBMM, GMSJ, PCM, NMLBP. Aprovação da versão final: HLPCS, FBMM, GMSJ, PCM, NMLBP. Responsabilidade pública pelo conteúdo do artigo: HLPCS, FBMM, GMSJ, PCM, NMLBP.

Conflito de Interesses: Os autores declaram não haver conflito de interesses. 